TRANSACTIONS OF THE

AMERICAN MATHEMATICAL SOCIETY

Volume 364, Number 4, April 2012, Pages 2087-2107

S 0002-9947(2011)05413-9

Article electronically published on December 1, 201

\title{
OBJECTIVE B-FIELDS AND A HITCHIN-KOBAYASHI CORRESPONDENCE
}

\begin{abstract}
SHUGUANG WANG
Abstract. A simple trick invoking objective B-fields is employed to refine the concept of characteristic classes for twisted bundles. Then the objective stability and objective Einstein metrics are introduced and a new HitchinKobayashi correspondence is established between them.
\end{abstract}

\section{INTRODUCTION}

Recently there has been intensive literature on gerbes starting from Brylinski's book [4, in which the Chern-Weil theory of gerbes was carried out. The geometry of abelian gerbes was further refined and clarified by Murray [21] and Chatterjee [6], and Hitchin [12] from two different viewpoints. These objects have found applications in physics for the descriptions of the so-called B-fields and the twisted K-theory, according to [1, 3, 17, 23] among many others. A real version of gerbes is presented in [27.

In one aspect gerbes are utilized to define twisted bundles where the usual cocycle condition fails to hold. The concept of twisted principal bundles was explicitly proposed in [18, 19] where their holonomy is studied, while the authors in [2, 22] introduced bundle gerbe modules in a different setting for the generalization of the index theory. In the context of algebraic geometry these are Azumaya bundles via the description of gerbes in terms of Brauer groups; see [20, 30, 5] for examples.

The first main purpose in our paper is to propose a new concept of Chern classes for twisted vector bundles as a refinement of existing definitions from [2, 5]. In fact the earlier definitions are not completely satisfactory in the sense that the one given in $[2$ is not quite topological because of the dependence on different curvings, while the definition in [5] involves an unspecified choice of a second twisted bundle. Our approach can be viewed as a balance of the two. To explain the key idea behind recognizing that the underlying gerbe $\mathcal{L}$ of a rank $m$-twisted vector bundle $E$ is an $m$-torsion, we select a trivialization $L$ of $\mathcal{L}^{m}$. To remove the dependence on the B-fields $B$ (namely curvings), we restrict ourselves to a special $B$ so that $m B$ is objective with respect to $L$. Given a twisted connection $D$ on $E$, twisted over a gerbe connection $\mathcal{A}$, we use their curvatures and $B$ together to define closed global Chern forms $\phi_{k}(E, L ; \mathcal{A}, B, D)$.

Theorem 0.1. The L-objective Chern classes of E, defined as

$$
c_{k}^{L}(E)=\left[\phi_{k}(E, L ; \mathcal{A}, B, D)\right] \in H^{2 k}(X, \mathbf{R}),
$$

Received by the editors July 30, 2009 and, in revised form, May 10, 2010 and June 15, 2010. 2010 Mathematics Subject Classification. Primary 53C07, 53C08, 57R20.

Key words and phrases. Objective Chern classes, Hermitian-Einstein metric, stable bundle, Hitchin-Kobayashi correspondence.

(C)2011 American Mathematical Society Reverts to public domain 28 years from publication 
are topological invariants of $E$. In other words, $c_{k}^{L}(E)$ are independent of the choices made for $D, \mathcal{A}, B$.

(The usage of the word "objective" throughout the paper was inspired by [6].) It seems likely that Theorem 0.1 will provide an alternative starting point to the current research [3, 2, 22, 30, concerning a host of index theorems and twisted K-theory.

With objective Chern classes and properly defined twisted subbundles, we further introduce objective stability. Moreover, by fixing a metric on $L$, we are able to characterize objective Einstein metrics so that we can formulate and generalize the Hitchin-Kobayashi correspondence as follows. (A possible statement as such was alluded to in [7].) The original correspondence for untwisted bundles was a corner-stone result that had been established in [16, 8, 25, 9,

Theorem 0.2. Suppose $E$ is a rank $m$ indecomposable holomorphic bundle twisted over a gerbe $\mathcal{L}$ and $L$ is a holomorphic trivialization of $\mathcal{L}^{m}$. Then $E$ is L-objective stable iff $E$ admits an $H$-objective Einstein metric for some Hermitian metric $H$ on $L$.

The theorem is new only if the base manifold has complex dimension at least 2. For the case of a complex curve, any gerbe $\mathcal{L}$ must be trivial; hence no twisting occurs. But one might still ask about stable bundles and Yang-Mills connections on non-orientable Riemann surfaces; compare 26] for example.

Our consideration of trivializations $L$ of $\mathcal{L}^{m}$ is rather natural and motivated in part by the lifting of $\mathrm{SO}(3)$-bundles. More precisely when an $\mathrm{SO}(3)$-bundle $S$ is $\operatorname{spin}^{c}$, lifting its structure group from $\mathrm{SO}(3)$ to $\mathrm{U}(2)$ one has the spinor bundle $E$ with determinant line bundle $\operatorname{det} E$. In general if $S$ is not $\operatorname{spin}^{c}$, one can only have a twisted $\mathrm{U}(2)$-bundle $E$ over a non-trivial gerbe $\mathcal{L}$. Then a trivialization $L$ of $\mathcal{L}^{2}$ is a replacement of the determinant. By using the twisted bundle theory in this paper, it seems possible to prove the orientability of instanton moduli spaces for $S$, generalizing Donaldson's results in Section 3 of [10] where $S$ is assumed to be $\operatorname{spin}^{c}$. Independent of the $\mathrm{SO}(3)$-vector bundle $S$, it is an interesting problem to investigate directly the instanton moduli space $M^{L}(E)$ on the twisted $\mathrm{U}(2)$-bundle $E$ with a fixed trivialization $L$. Perhaps one could further extract Donaldson type invariants. In terms of algebraic geometry, this suggests the consideration of the moduli space of $L$-objective stable bundles. We hope to pursue these separate issues in a future work.

Here is a brief description of the paper. After reviewing and setting up notation for gerbes and so on in Section 1, we develop the objective Chern-Weil theory and prove Theorem 0.1 in Section 2. The fundamental concepts of objective subbundles, Einstein metrics and stability are laid out in Sections 3 and 4 . Finally, Sections 5 and 6 are devoted to the proofs of the two directions in Theorem 0.2.

\section{Review of Gerbes, B-Fields AND twisted BUndLes}

All vector bundles and differential forms will be defined over the complex field C unless otherwise indicated.

To set up notation, first recall the definition of gerbes from [6, 12]. Let $X$ be a smooth manifold $X$ with an open cover $\left\{U_{i}\right\}$. A gerbe $\mathcal{L}=\left\{L_{i j}\right\}$ consists of line bundles $L_{i j} \rightarrow U_{i} \cap U_{j}$ with given isomorphisms

$$
L_{i j}=L_{j i}^{-1}, \quad L_{i j} \otimes L_{j k}=L_{i k}
$$


on their common domains of definition. Using a good cover or a refinement of the cover if necessary, we can assume $L_{i j}$ are trivial bundles. Choose trivializations $\xi_{i j}$ for $L_{i j}$. They should be compatible with (11) in that

$$
\xi_{i j}=\xi_{j i}^{-1}, \quad \xi_{i j} \otimes \xi_{j k}=z_{i j k} \xi_{i k},
$$

where $\underline{z}=\left\{z_{i j k}\right\}$ forms a Cech 2-cycle of the sheaf $\underline{\mathbf{C}}^{*}$ of nowhere vanishing complex functions on $X$. One imposes that $\underline{z}$ be co-closed: $\delta \underline{z}=1$.

Thus the gerbe class $[\mathcal{L}]:=[\underline{z}] \in \check{H}^{2}\left(X, \underline{\mathbf{C}}^{*}\right)$ is defined. Two gerbes are stable isomorphic if there is a common refinement such that they become the same after tensoring with line bundles of the form $\left\{L_{i}^{*} \otimes L_{j}\right\}$ over $\left\{U_{i} \cap U_{j}\right\}$. This is equivalent to their gerbe classes being the same. As in the case of bundles, it is often convenient not to distinguish a gerbe $\mathcal{L}$ from its class $[\mathcal{L}]$. One can view $[\mathcal{L}] \in H^{3}(X, \mathbf{Z})$ according to the natural isomorphism $\check{H}^{2}\left(X, \underline{\mathbf{C}}^{*}\right) \rightarrow H^{3}(X, \mathbf{Z})$, which comes from the short exact sequence $\mathbf{Z} \rightarrow \underline{\mathbf{C}} \rightarrow \underline{\mathbf{C}}^{*}$. Gerbes form an Abelian group under the tensor product.

From (11), one has a trivialization $s_{i j k}$ of $L_{i j k}:=L_{i j} \otimes L_{j k} \otimes L_{k i}$ as part of the gerbe definition. In [6, 12, a gerbe connection $\left\{A_{i j}, B_{i}\right\}$ on $\mathcal{L}$ consists of two parts: connections $A_{i j}$ on $L_{i j}$ and complex 2 -forms $B_{i}$ on $U_{i}$, such that 1) $s_{i j k}$ is a covariant constant trivialization of $L_{i j k}$ under the induced product connection using $A_{i j}$, and 2) $B_{j}-B_{i}=F_{A_{i j}}$ on $U_{i} \cap U_{j}$ for the curvature of $A_{i j}$. However, in this paper we will separate them and call $\mathcal{A}=\left\{A_{i j}\right\}$ alone a gerbe connection and give a more prominent role to $B=\left\{B_{i}\right\}$, which we call a ( $\mathcal{A}$-compatible) $B$ field from its physics interpretation. The closed local 3-form $\left\{d B_{i}\right\}$ can be patched together to yield a global form $G$, called the gerbe curvature of the pair $\mathcal{A}, B$. The class $[G] \in H^{3}(X, \mathbf{R})$ is the real image of $[\mathcal{L}] \in H^{3}(X, \mathbf{Z})$, hence independent of the choice of $\mathcal{A}, B$. Moreover, every representative of the real image can be realized by some B-field and connection. Here is a collection of useful facts to recall:

Proposition 1.1. The following are equivalent:

1) $\mathcal{L}$ is a torsion, i.e. $m[\mathcal{L}]=0 \in H^{3}(X, \mathbf{Z})$ for some integer $m$.

2) Under a suitable cover of $X,[\mathcal{L}]$ can be represented by a Čech 2-cycle $\underline{z}=$ $\left\{z_{i j k}\right\}$ such that $z_{i j k}^{m}=1$ for some fixed $m$. (Hence $\underline{z}$ must be locally constant.)

3) The real gerbe class of $\mathcal{L}$ is trivial, namely the image $[\mathcal{L}]=0$ in $H^{3}(X, \mathbf{R})$.

4) $\mathcal{L}$ is flat, i.e. there exists a gerbe connection $\mathcal{A}$ with a compatible $B$-field $B$ such that the curvature 3 -form $G=0$.

Next suppose $\mathcal{L}$ is trivial, i.e. its class $[\mathcal{L}]=0 \in \check{H}^{2}\left(X, \underline{\mathbf{C}}^{*}\right)$. Then $\underline{z}$ is a coboundary of a Čech 1-cocycle, which leads to a trivialization $L=\left\{L_{i}\right\}$ of $\mathcal{L}$, namely line bundles $L_{i}$ on $U_{i}$ such that $L_{j}=L_{i} \otimes L_{i j}$ when restricted to $U_{i} \cap U_{j}$. Given a second trivialization $L^{\prime}=\left\{L_{i}^{\prime}\right\}$, we have a global line bundle $\ell$ on $X$, called the difference bundle of $L$ and $L^{\prime}$. We will denote it as $\ell=L \ominus L^{\prime}$. A trivialization of a gerbe connection $\mathcal{A}$ is a family of connections $A=\left\{A_{i}\right\}$ on $\left\{L_{i}\right\}$ subject to an analogous condition. Since the 1-form sheaf is a fine sheaf, trivializations always exist for any gerbe connection but are not unique. Given a second trivializing connection $A^{\prime}$ on $L^{\prime}$, we have a difference connection $A \ominus A^{\prime}$ on the bundle $\ell$.

Given a trivializing connection $A$ of $\mathcal{A}$, the collection of local curvature forms $\left\{F_{A_{i}}\right\}$ is a B-field compatible with $\mathcal{A}$; but not every compatible B-field has this form (i.e. from some trivializing connection of $\mathcal{A}$ ). Given a compatible B-field $B$ and a trivializing connection $A$, the difference $\epsilon:=B_{i}-F_{A_{i}}$ is a global 2-form on 
$X$. This error form $\epsilon$ is essential in defining the gerbe holonomy of $\mathcal{A}$. Following [ 6 ] we call $B$ objective if $\epsilon=0$ for some $A$. A necessary condition is that $B_{i}$ be closed. Suppose this is so. Then the error form $\epsilon$ with any trivializing connection is also closed and hence gives rise to a class $[\epsilon] \in H^{2}(X, \mathbf{R})$. In this language, objective B-fields are exactly those closed B-fields such that the error class $[\epsilon]$ lives in the lattice $H^{2}(X, \mathbf{Z}) \subset H^{2}(X, \mathbf{R})$. Here of course a gerbe connection $\mathcal{A}$ has been fixed throughout.

Now is a good time to set out the following guide for our notation in the paper.

Remark 1.2. We will continue using script letters such as $\mathcal{L}, \mathcal{A}$ for things defined on intersections of open sets $\left\{U_{i}\right\}$ and reserve standard block letters $L, B$, $A$, etc. for objects defined on open sets themselves.

A trivialization $L=\left\{L_{i}\right\}$ of $\mathcal{L}$ can also be viewed as a twisted line bundle over $\mathcal{L}$. In general, given an arbitrary gerbe $\mathcal{L}$, an $\mathcal{L}$-twisted vector bundle $E=\left\{E_{i}\right\}$ on $X$ consists of a collection of local bundles of the same rank such that $E_{j}=$ $E_{i} \otimes L_{i j}$ on $U_{i} \cap U_{j}$. We will also say that $E$ is over the twisting gerbe $\mathcal{L}$. (Twisted principal bundles were first defined in [18.) In a similar spirit, one defines a twisted connection $D=\left\{D_{i}\right\}$ on $E$ over a gerbe connection $\mathcal{A}$. We will adopt the following convenient notation to indicate the twisting gerbe $\mathcal{L}$ and the twisting connection $\mathcal{A}$ :

$$
E \prec \mathcal{L}, D \prec \mathcal{A} \text {. }
$$

Twisted bundles/connections are not necessarily mysterious: simply put, these are so defined that their projectivizations are just regular fiber bundles and connections, respectively. For any 1-dimensional vector space $V, V \otimes V^{*}$ carries a canonical basis. It follows that the local endomorphism bundles $\left\{\operatorname{End}\left(E_{i}\right)\right\}$ naturally fit together to produce a global vector bundle on $X$, in view of $\operatorname{End}\left(E_{i}\right)=E_{i} \otimes E_{i}^{*}$. We denote it by $\operatorname{End}(E)$. Note that the wedge product twisted bundle is over $\mathcal{L}^{r}$ :

$$
\bigwedge^{r} E=\left\{\bigwedge^{r} E_{i}\right\} \prec \mathcal{L}^{r}=\left\{\bigotimes^{r} L_{i j}\right\} .
$$

In particular, $\operatorname{det} E$ gives rise to a trivialization of $\mathcal{L}^{m}$, where $m=\operatorname{rank} E$. In other words, the underlying gerbe $\mathcal{L}$ of $E$ must be an $m$-torsion.

Other operations can be introduced as well: one has the dual $E^{*} \prec \mathcal{L}^{-1}$. If $E^{\prime} \prec \mathcal{L}^{\prime}$, then $E \otimes E^{\prime} \prec \mathcal{L} \otimes \mathcal{L}^{\prime}$. But for $E \oplus E^{\prime}$, one must impose $\mathcal{L}=\mathcal{L}^{\prime}$; then $E \oplus E^{\prime} \prec \mathcal{L}$.

\section{ObJective Chern Classes for twisted Bundles}

We now develop a new version of Chern-Weil theory for rank $m$-twisted vector bundles. Fix a gerbe $\mathcal{L}$ with connection $\mathcal{A}$ and B-field $B$. Assume $\mathcal{L}$ is an $m$-torsion. By Proposition 1.1, we can choose $B$ to be flat so that all $B_{i}$ are closed 2-forms. For a twisted rank $m$-vector bundle $E \prec \mathcal{L}$ with connection $D \prec \mathcal{A}$, it is easy to check that B-twisted curvature of $D$ :

$$
\widetilde{F}_{D}=F_{D_{i}}-B_{i} I
$$

is a global section of $\Omega^{2}(\operatorname{End}(E))$. In order to get the expected topological results we will need to place a crucial restriction on $B$.

Theorem 2.1. Consider an $m$-torsion gerbe $\mathcal{L}$ with connection $\mathcal{A}$ and $B$-field $B$. Let $L=\left\{L_{i}\right\}$ be a trivialization of $\mathcal{L}^{m}$ and $A=\left\{A_{i}\right\}$ be that of $\mathcal{A}^{m}$. Assume the $B$-field $m B$ on $\mathcal{L}^{m}$ to be objective: $m B=\left\{F_{A_{i}}\right\}$ (one may call $B$ relative objective). 
Let $E$ be a rank $m$ twisted vector bundle over $\mathcal{L}$ and $D=\left\{D_{i}\right\}$ be a twisted connection over $\mathcal{A}$. Introduce the total Chern form

$$
\phi(E, L ; \mathcal{A}, B, D)=\operatorname{det}\left(I+\frac{i}{2 \pi} \widetilde{F}_{D}\right) .
$$

1) Each homogeneous part $\phi_{k}(E, L ; \mathcal{A}, B, D)$ is a closed $2 k$ form on $X$.

2) The class $\left[\phi_{k}\right] \in H^{2 k}(X, \mathbf{R})$ is independent of the choices of $\mathcal{A}, B, D$.

3) Suppose $L^{\prime}=\left\{L_{i}^{\prime}\right\}$ is a second trivialization of $\mathcal{L}^{m}$ and $A^{\prime}=\left\{A_{i}^{\prime}\right\}$ is a trivialization of $\mathcal{A}^{m}$ on $L^{\prime}$. Let $B^{\prime}$ be the $B$-field on $\mathcal{L}$ such that $m B^{\prime}=\left\{F_{A_{i}^{\prime}}\right\}$. Then the following holds:

$$
\left[\phi_{k}\left(E, L^{\prime} ; \mathcal{A}, B^{\prime}, D\right)\right]=\sum_{i=0}^{k} \frac{C(m-i, k-i)}{m^{k-i}}\left[\phi_{i}(E, L ; \mathcal{A}, B, D)\right] c_{1}(\ell)^{k-i},
$$

where $\ell=L \ominus L^{\prime}$ is the difference line bundle of $L, L^{\prime}$. (Here and below $C(r, s)$ stands for the combinatorial expression $\frac{r !}{s !(r-s) !}$.)

Proof. 1) Following the standard Chern-Weil theory (for example from Chapter III of [29]), it suffices to show that a Bianchi type identity still holds:

$$
d \widetilde{F}_{D}=\left[\widetilde{F}_{D}, \widetilde{\theta}\right]
$$

for some $\widetilde{\theta} \in \Omega^{1}(\operatorname{End}(E))$ on any small open set $V \subset U_{i}$. To prove the identity, if $V$ is small enough, there is a line bundle $K \rightarrow V$ such that $L_{i}=K^{m}$. Set $A_{i}=G^{m}$ for some unique connection $G$ on $K$. Then $B_{i}=\frac{1}{m} F_{A_{i}}=F_{G}$. It follows that

$$
\widetilde{F}_{D}=F_{D_{i}}-F_{G} I=F_{\widetilde{D}}
$$

for the curvature of the tensor product connection $\widetilde{D}=D \otimes G^{-1}$ on $E_{i} \otimes K^{-1}$. Now the standard Bianchi identity for $\widetilde{D}$ yields (3), where $\widetilde{\theta}$ is the connection matrix of $\widetilde{D}$ under a local frame.

2) Note that $\mathcal{A}$ and $B$ are both determined uniquely by $A$ (although $\mathcal{L}$ is not so by $L)$. It is enough to show more generally that $\left[\phi_{k}\right]$ is independent of the choices of $D, A$. For a second pair of choices $D^{\prime}=\left\{D_{i}^{\prime}\right\}, A^{\prime}=\left\{A_{i}^{\prime}\right\}$, consider the one-parameter families $D(t)=\left\{t D_{i}+(1-t) D_{i}^{\prime}\right\}, A(t)=\left\{t A_{i}+(1-t) A_{i}^{\prime}\right\}$. Then $A(t)$ determines families of gerbe connections $\mathcal{A}(t)$ and objective B-fields $B(t)$ on $\mathcal{L}$. Moreover, $D(t)$ is a twisted connection over $\mathcal{A}(t)$ for each $t$.

Set the family $\widetilde{F}(t)=F_{D(t)}-B(t) I$. On a small enough open set $V \subset U_{i}$, let $L_{i}=K^{m}$ as above and $G(t)$ be the family of connections on $K$ determined by $A_{i}(t)$. Then $\widetilde{F}(t)=F_{\widetilde{D}(t)}$, the curvature of the tensor product connections $\widetilde{D}(t)=D(t) \otimes G(t)^{-1}$ on $E_{i} \otimes K^{-1}$. Thus

$$
\widetilde{F}(t)=d \widetilde{\theta}(t)+\widetilde{\theta}(t) \wedge \widetilde{\theta}(t)
$$

for the connection matrix $\widetilde{\theta}(t)$ of $\widetilde{D}(t)$ under a frame. Consequently,

$$
\dot{\widetilde{F}}=\dot{d \vec{\theta}}+[\dot{\widetilde{\theta}}, \widetilde{\theta}] .
$$

As in the standard Chern-Weil theory [29, the above formula together with the Bianchi formula (3) shows that

$$
\phi_{k}\left(E, L ; \mathcal{A}^{\prime}, B^{\prime}, D^{\prime}\right)-\phi_{k}(E, L ; \mathcal{A}, B, D)
$$

is an exact form. Hence $\left[\phi_{k}\left(E, L ; \mathcal{A}^{\prime}, B^{\prime}, D^{\prime}\right)\right]=\left[\phi_{k}(E, L ; \mathcal{A}, B, D)\right]$. 
3) Here it is important to be able to keep the same gerbe connection $\mathcal{A}$ on $\mathcal{L}$ and hence the same twisted connection $D$ on $E$. In other words a different trivialization $L^{\prime}$ will only impact on the B-field via $A^{\prime}$. Now

$$
F_{D}-B^{\prime} I=F_{D}-B I+\left(B-B^{\prime}\right) I=F_{D}-B I+\frac{1}{m} F_{\alpha} I,
$$

where $\alpha$ is the difference connection of $A, A^{\prime}$ on $\ell$. Under a frame, write $F_{D}-B I$ as a matrix $\left(F_{j}^{i}\right)$ of 2 -forms. Then $F_{D}-B^{\prime} I$ is given by the matrix $\left(F_{j}^{i}+\frac{1}{m} \delta_{j}^{i} F_{\alpha}\right)$. Applying the classical formula to $\operatorname{det}\left(I+F_{D}-B I\right)$, one has

$$
\phi_{k}(E, L ; \mathcal{A}, B, D)=\frac{1}{(2 \pi i)^{k} k !} \sum \delta_{i_{1} \cdots i_{k}}^{j_{1} \cdots j_{k}} F_{j_{1}}^{i_{1}} \wedge \cdots \wedge F_{j_{k}}^{i_{k}} .
$$

Doing the same for $\operatorname{det}\left(I+F_{D}-B^{\prime} I\right)$ will give

$$
\begin{aligned}
& \phi_{k}\left(E, L^{\prime} ; \mathcal{A}, B^{\prime}, D\right) \\
& \quad=\frac{1}{(2 \pi i)^{k} k !} \sum \delta_{i_{1} \cdots i_{k}}^{j_{1} \cdots j_{k}}\left(F_{j_{1}}^{i_{1}}+\frac{1}{m} \delta_{j_{1}}^{i_{1}} F_{\alpha}\right) \wedge \cdots \wedge\left(F_{j_{k}}^{i_{k}}+\frac{1}{m} \delta_{j_{k}}^{i_{k}} F_{\alpha}\right) .
\end{aligned}
$$

These formulas together will give us the desired result for their classes after noting $c_{1}(\ell)=\left[F_{\alpha}\right]$.

In particular, if $L^{\prime}$ is flat equivalent to $L$, namely if $\ell$ is flat, then $c_{1}(\ell)=0 \in$ $H^{2}(X, \mathbf{R})$ and the class $\left[\phi_{k}\right]$ remains the same. This is the case if $L^{\prime}$ is isomorphic to $L$ (i.e. $\ell$ is trivial). Thus it makes sense to introduce:

Definition 2.2. Suppose $E \prec \mathcal{L}$ is a rank $m$ twisted bundle and $L \prec \mathcal{L}^{m}$ is a trivialization. The $L$-objective Chern class of $E$ is defined as follows:

$$
c_{k}^{L}(E)=\left[\phi_{k}(E, L ; \mathcal{A}, B, D)\right] \in H^{2 k}(X, \mathbf{R}),
$$

where $\mathcal{A}$ is any gerbe connection on $\mathcal{L}, D$ is any twisted connection on $E$ and $B$ is a B-field compatible with $\mathcal{A}$ such that $m B$ is objective.

Remark 2.3. Alternatively one could define a twisted bundle as a pair $(E, L)$ where $E \prec \mathcal{L}$ and $L \prec \mathcal{L}^{m}$ in our notation. Then one could define the Chern classes for $(E, L)$ without the cumbersome prefix " $L$-objective". For us the advantage of separating $L$ from $E$ is that we can better illustrate the dependence on $L$ as shown in the next corollary.

Corollary 2.4. Suppose $E \prec \mathcal{L}$ and $L, L^{\prime} \prec \mathcal{L}^{m}$. Then

$$
c_{k}^{L^{\prime}}(E)=\sum_{i=0}^{k} \frac{C(m-i, k-i)}{m^{k-i}} c_{i}^{L}(E) c_{1}(\ell)^{k-i}
$$

in terms of the difference line bundle $\ell=L \ominus L^{\prime}$. In particular,

$$
c_{1}^{L^{\prime}}(E)=c_{1}^{L}(E)+c_{1}(\ell) .
$$

Recall $\bigwedge^{r} E \prec \mathcal{L}^{r}$ for any integer $r \leq m$. Setting $w=C(m-1, r-1)$, one also has

$$
c_{1}^{L^{w}}\left(\bigwedge^{r} E\right)=w c_{1}^{L}(E)
$$

In particular, $c_{1}^{L}(\operatorname{det} E)=c_{1}^{L}(E)$, as one might expect. 
Proof. The first formula is a translation of part 3) of Theorem 2.1. To show the third formula, note that $\operatorname{rank} \bigwedge^{r} E=C(m, r)$ and

$$
r C(m, r)=m C(m-1, r-1)=m w .
$$

Hence $L^{w}$ is a trivialization of $\mathcal{L}^{r C(m, r)}$; the latter carries the B-field $C(m, r) r B$. Let $\wedge D$ denote the induced twisted connection on $\wedge^{r} E$. One then has

$$
\begin{aligned}
c_{1}^{L^{w}}\left(\bigwedge^{r} E\right) & =\operatorname{tr} F_{\wedge D}-C(m, r) r B \\
& =w \operatorname{tr} F_{D}-m w B=w\left(\operatorname{tr} F_{D}-m B\right) \\
& =w c_{1}^{L}(E),
\end{aligned}
$$

delivering us the desired formula.

Remark 2.5. One could in particular use the special trivialization $\operatorname{det} E=\bigwedge^{m} E \prec$ $\mathcal{L}^{m}$ to introduce $c_{k}(E):=c_{k}^{\operatorname{det} E}(E)$. For any other trivialization $L \prec \mathcal{L}^{m}$, the previous corollary then yields

$$
c_{k}^{L}(E)=\sum_{i=0}^{k} \frac{C(m-i, k-i)}{m^{k-i}} c_{i}(E) c_{1}(\ell)^{k-i}
$$

using the difference bundle $\ell=\operatorname{det} E \ominus L$. Note that $c_{1}(E)=0$ and the formula above implies that $c_{1}^{L}(E)=c_{1}(\ell)$ is always an integer class.

The following example from gauge theory has been a useful guide for us and will also show why it is important to expand $c_{k}(E)$ to $c_{k}^{L}(E)$ with the incorporation of trivializations other than $\operatorname{det} E$.

Example 2.6. Let $Q$ be an $\mathrm{SO}(3)$-vector bundle on $X$. Suppose $Q$ is $\operatorname{spin}^{c}$ so that $w_{2}(Q)$ has integral lifts in $H^{2}(X, \mathbf{Z})$. Fix such a lift $L$ viewed as a line bundle as well as a connection $A$ on it. Then $Q$ together with connection $\nabla$ will lift to a unique $\mathrm{U}(2)$-vector bundle $\widetilde{Q}$ with connection $\widetilde{\nabla}$ such that $\operatorname{det}(\widetilde{Q})=L$, $\operatorname{det} \widetilde{\nabla}=A$. We can interpret $\widetilde{Q}, \widetilde{\nabla}$ and especially the Chern classes $c_{k}(\widetilde{Q})$ in terms of our twisted vector bundle theory. For this purpose choose any open cover $\left\{U_{i}\right\}$ of $X$ such that $\left.L\right|_{U_{i}}$ has a square root $K_{i}:=\sqrt{L}$ on $U_{i}$. Thus $K=\left\{K_{i}\right\}$ is a trivialization of the gerbe $\mathcal{L}=\left\{L_{i j}\right\}$ where $L_{i j}=K_{j} \otimes K_{i}^{*}$ by definition. (The gerbe $\mathcal{L}$, first observed in [28, can be formally viewed as being given by $\sqrt{L}$, namely $\mathcal{L}$ is the obstruction to the existence of a global squared root of $L$. Similarly there is a gerbe given by the $n$-th root $\sqrt[n]{L}$. More on real gerbes can be found in [26.) Moreover, $A$ leads to a unique twisted connection $\widetilde{A}=\left\{\widetilde{A}_{i}\right\} \prec \mathcal{A}$ on $K$, where $\mathcal{A}=\left\{A_{i j}\right\}$ and $A_{i j}$ is the tensor product connection $\widetilde{A}_{j} \otimes \widetilde{A}_{i}^{*}$ on $L_{i j}$. Now on each $U_{i}$, lift $\widetilde{Q}$ to an $\operatorname{SU}(2)$ vector bundle $E_{i}$ so that $E=\left\{E_{i}\right\}$ is a twisted bundle over $\mathcal{L}$. Then $\nabla$ lifts to a unique twisted connection $D=\left\{D_{i}\right\} \prec \mathcal{A}$ on $E$. Now one can easily check that

$$
\widetilde{Q}=E_{i} \otimes K_{i}, \widetilde{\nabla}=D_{i} \otimes \widetilde{A}_{i}, c_{k}(\widetilde{Q})=c_{k}^{L}(E),
$$

where $L=K^{2} \prec \mathcal{L}^{2}$ is viewed as a trivialization of $\mathcal{L}^{2}$. (Here $K_{i}^{2}$ is global since $\mathcal{L}^{2}$ is trivial as a Čech cycle, not just as a Čech class.)

When $Q$ is not $\operatorname{spin}^{c}$, the line bundle $L$ will not exist any longer. Nonetheless, $Q$ still lifts locally to a twisted SU(2) bundle $E=\left\{E_{i}\right\}$ over some gerbe $\mathcal{L}$. (The nontrivial gerbe class $[\mathcal{L}]$ equals $W_{3}(Q) \in H^{3}(X, \mathbf{Z})$.) The essence of our theory above is to replace $L$ with a trivializing twisted bundle of $\mathcal{L}^{2}$, and such a trivialization always exists! More generally, the lesson for our entire paper is this: given twisted 
data such as $E, D$ that are defined on local open sets only, one should couple them with objective gerbe data in order to get global data on $X$.

Remark 2.7. We now compare our Definition 2.2 with several existing definitions of Chern classes in the literature.

1) To get the topological invariance, it was important and indeed necessary for us to impose the condition that $m B$ be objective in Theorem 2.1. For an arbitrary flat B-field $B$, without invoking $L$ or assuming $m B$ to be objective, one can still prove that $\phi(E ; \mathcal{A}, B, D)=\operatorname{det}\left(I+\frac{1}{2 \pi i} \widetilde{F}_{D}\right)$ is closed. (Indeed one has a Bianchi identity directly from the following computation:

$$
d \widetilde{F}_{D}=d\left(F_{D_{i}}-B_{i} I\right)=d F_{D_{i}}=\left[F_{D_{i}}, \theta\right]=\left[\widetilde{F}_{D}, \theta\right],
$$

where $\theta$ is the connection matrix of $D_{i}$ under a local frame and the last equation follows from $\left[B_{i} I, \theta\right]=0$ since $B_{i}$ is a 2-form.) One could then try to define the Chern class of $E$ as $\widetilde{c}_{k}(E)=\left[\phi_{k}(E ; \mathcal{A}, B, D)\right]$. In terms of bundle gerbe modules, this seems to correspond to the Chern character using an arbitrary curving $f$ in Section 6.3 of Bouwknegt et al. 2. However such an expanded definition in either place has the issue that $\widetilde{c}_{k}(E)$ is not well defined but depends on the choices made for $\mathcal{A}, B, D$. Without invoking $L$, it is not possible to characterize such dependence. The indeterminacy is essentially due to the fact that the compatibility between $B$ and $\mathcal{A}$ only mildly constrains $B$ by $\mathcal{A}$.

2) In [5], Căldăraru proposed to define the Chern classes $c^{W}(E)$ of $E$ as the regular Chern classes $c(E \otimes W)$, where $W$ is a fixed twisted vector bundle over $\mathcal{L}^{-1}$. The main problem in this approach is that no natural choices were given for $W$ in applications. In comparison, our choices $L$ as trivializations of $\mathcal{L}^{m}$ are pertinent and natural, in view of the fact that $\mathcal{L}$ is an $m$-torsion as the underlying gerbe of $E$. Compare also with [13, where $B$ is taken to be a global closed 2form on $X$. However, that is possible only because the gerbe $\mathcal{L}$ is trivial in their applications for K3-complex surfaces $X$.

3) When the gerbe connection $\mathcal{A}$ is flat, namely all $F_{A_{i j}}=0$, one can take $B_{i}=0$ for all $i$. (This is a constraint on $L$.) Thus $\left\{F_{D_{i}}\right\}$ is already a global section of $\operatorname{End}(E)$ and our Chern classes $c_{k}^{L}(E)$ are given by $\operatorname{det}\left(I+\frac{1}{2 \pi i} F_{D_{i}}\right)$. Passing over to bundle gerbe modules, this special case should correspond to the Chern classes in $[20,22]$.

It is an interesting problem to apply the objective Chern-Weil theory to various index theorems and twisted K-theory, which we hope to return to in a future work.

\section{Objective sections And subBundles of TWisted BundLES}

We begin by the following definition; the second part seems to be brand new.

Definition 3.1. Suppose $E \prec \mathcal{L}$ and $L \prec \mathcal{L}^{m}$. As in formula (2), $\mathcal{L}$ comes with nowhere vanishing local sections $\xi=\left\{\xi_{i j}\right\}$.

1) A twisted section of $E$ is a collection of local sections $s=\left\{s_{i}\right\}$ of $E_{i}$ satisfying $s_{j}=s_{i} \otimes \xi_{i j}$ under the identification $E_{j}=E_{i} \otimes L_{i j}$.

2) Note that $L^{-1} \prec \mathcal{L}^{-m}$ and the latter carries local sections $\xi^{-m}=\left\{\xi_{i j}^{-m}\right\}$. Suppose $\xi^{-m}$ is objective, namely there are local sections $t=\left\{t_{i}\right\}$ of $L^{-1}$ such that $t_{j}=t_{i} \otimes \xi_{i j}^{-m}$. We will rephrase $s$ as a $t$-objective section of $E$ in this context, in order to emphasize the relevance of $\left\{t_{i}\right\}$. 
The main point of introducing objective sections is that $s_{i} \otimes \sqrt[m]{t_{i}}$ will be a global section, namely $s_{i} \otimes \sqrt[m]{t_{i}}=s_{j} \otimes \sqrt[m]{t_{j}}$ on the overlap, whenever $\sqrt[m]{t_{i}}$ is properly defined. Of course here $t$ is just a twisted section of $L^{-1}$ over $\mathcal{L}^{-m}$. We use $L^{-1}$ instead of $L$, since $t_{i}$ may vanish and so $t_{i}^{-1}$ may not exist.

Since generally $\xi^{-m}$ is not objective, the existence of $t$ does constrain $s$ via $\xi$. In fact one readily checks that existence of a nowhere vanishing $t$ is equivalent to the fact that the Čech 2-cycle $\underline{z}=\left\{z_{i j k}\right\}$ of $\mathcal{L}$ has order $m$ : $\underline{z}^{m}=1$. By Proposition 1.1. such a 2-cycle exists only on special covers.

From these sections we can define twisted bundle homomorphisms in a rather formal way.

Definition 3.2. Suppose $F \prec \mathcal{K}, E \prec \mathcal{L}$ are twisted vector bundles of ranks $n, m$, respectively. Let $K \prec \mathcal{K}^{n}, L \prec \mathcal{L}^{m}$ be chosen trivializations.

1) A twisted homomorphism $f: F \rightarrow E$ is just any twisted section of the bundle $F^{*} \otimes E \prec \mathcal{K}^{*} \otimes \mathcal{L}$.

2) From a twisted homomorphism $g: L^{n} \rightarrow K^{m}$, one then has a $g$-objective homomorphism $f$. Here $L^{n} \prec \mathcal{L}^{m n}, K^{m} \prec \mathcal{K}^{n m}$ are the associated twisted bundles. (Note that $g$ maps in the opposite direction as $f$.)

More clearly, the gerbes $\mathcal{K}, \mathcal{L}$ come with local trivializations $\eta_{i j}, \xi_{i j}$ as in equation (2). They give rise to bundle isomorphisms $\chi_{i j}: K_{i j} \rightarrow L_{i j}$ by sending $\eta_{i j}$ to $\xi_{i j}$. Then $f=\left\{f_{i}: F_{i} \rightarrow E_{i}\right\}$ should satisfy

$$
f_{j}=f_{i} \otimes \chi_{i j} \text { on } F_{j}=F_{i} \otimes K_{i j} .
$$

Moreover, $g=\left\{g_{i}: L_{i}^{n} \rightarrow K_{i}^{m}\right\}$ and $g_{j}=g_{i} \otimes \chi_{i j}^{-m n}$ on $L_{j}^{n}=L_{i}^{n} \otimes L_{i j}^{m n}$, where $\chi_{i j}^{-1}$ is the inverse of $\chi_{i j}$. For a $g$-objective homomorphism $f, f \otimes \sqrt[m n]{g}$ will be a global homomorphism if suitably defined.

In particular, we can define a twisted or $g$-objective subbundle $F \prec \mathcal{K}$ of $E \prec \mathcal{L}$. However, in this approach, it is unclear how $\mathcal{K}$ should be tied to $\mathcal{L}$. Obviously one cannot always choose the same gerbe $\mathcal{K}=\mathcal{L}$ for all subbundles. For example, for a twisted line bundle $F$, the gerbe $\mathcal{K}$ must be trivial. Thus if $\mathcal{L}$ is non-trivial, then $E$ does not allow any twisted line subbundles if one insists on using the same gerbe.

To handle the issue more decisively, we shall adopt a different approach for twisted subbundles and make a comparison with the above general approach at the end. We start by the following arithmetic result. All integers are assumed to be positive.

Lemma 3.3. Given two integers $n \leq m$, let $T=T_{n, m}$ denote the set of integers $d$ such that each nd is divisible by $m$. Then $T$ contains two special elements $m_{*}=$ $\frac{m}{\operatorname{gcd}(m, n)}$ and $m^{*}=C(m, n)$. Moreover, $m_{*}$ is the common divisor and hence the smallest in $T$.

The last statement is proved by factoring $m, n$ into products of primes. (Of course $m \in T$ and $m_{*} \leq m \leq m^{*}$, hence the notation.) The key issue in defining twisted subbundles is to spell out what should be the underlying twisting gerbes.

Definition 3.4. Suppose $E=\left\{E_{i}\right\} \prec \mathcal{L}$ is a rank $m$ twisted vector bundle. An $\mathcal{L}^{d}$-twisted subbundle $F$ of rank $n$ is a collection of subbundles $\left\{F_{i}\right\}$ of $\left\{E_{i}\right\}$ such that $F$ by itself is a twisted bundle over $\mathcal{L}^{d}$, where $d \in T_{n, m}$.

The definition makes sense since each $\mathcal{L}^{d}$ is indeed an $n$-torsion gerbe by the definition of $T$. If $n=1$, then $m_{*}=m^{*}=m$ and any twisted line subbundle 
in this case is over the gerbe $\mathcal{L}^{m}$, hence a trivialization of $\mathcal{L}^{m}$. For other $d \in T$, an $\mathcal{L}^{d}$-twisted line subbundle of $E$ may be subject to a larger gerbe $\mathcal{L}^{m s}$ for some $s$. The proposition below exhibits certain compatibilities with wedge product and subbundle operations.

Proposition 3.5. Suppose $E \prec \mathcal{L}$ is a rank $m$ twisted bundle and $F, W$ are twisted bundles of ranks $n, r$.

1) If $F \subset E$ is an $\mathcal{L}^{m^{*}}$-twisted subbundle, then the wedge product $\bigwedge^{k} F$ is also an $\mathcal{S}^{m^{*}}$-twisted subbundle of $\bigwedge^{k} E$, where $\mathcal{S}=\mathcal{L}^{k}$ is the twisting gerbe of $\bigwedge^{n} E$.

2) If $F \subset E, W \subset F$ are, respectively, $\mathcal{L}^{d}$ and $\mathcal{G}^{d^{\prime}}$-twisted subbundles, where $d \in T_{n, m}, d^{\prime} \in T_{r, n}$ and $\mathcal{G}=\mathcal{L}^{d}$ is the twisting gerbe of $F$, then $W \subset F$ is a $\mathcal{L}^{\text {dd' }}$-twisted subbundle.

Proof. 1) Since $\bigwedge^{k} F$ is over the gerbe $\left(\mathcal{L}^{m^{*}}\right)^{k}=\mathcal{S}^{m^{*}}$, to show $\bigwedge^{k} F \subset \bigwedge^{k} E$ is an $\mathcal{S}^{m^{*}}$-twisted subbundle, one only needs to check $m^{*} \in T_{n^{\prime}, m^{\prime}}$, where $n^{\prime}=$ $\operatorname{rank}\left(\bigwedge^{k} F\right), m^{\prime}=\operatorname{rank}\left(\bigwedge^{k} E\right)$. This in turn follows from the identity

$$
C(n, k) C(m, n)=C(m-k, n-k) C(m, k) .
$$

(However $m^{*} \neq\left(m^{\prime}\right)^{*}$, the latter being $C\left(m^{\prime}, n^{\prime}\right)$ by definition.)

2) This amounts to the natural map

$$
\begin{array}{ccc}
T_{n, m} \times T_{r, n} & \longrightarrow & T_{r, m} \\
\left(d, d^{\prime}\right) & \mapsto & d d^{\prime},
\end{array}
$$

which can be confirmed directly.

Note that statement 1 ) is false if $\mathcal{L}^{m^{*}}$ is replaced by $\mathcal{L}^{m_{*}}$. Namely, for an $\mathcal{L}^{m_{*}}$ twisted subbundle $F \subset E$, the wedge product $\bigwedge^{k} F \prec \mathcal{S}^{m_{*}}$ may not be a twisted subbundle of $\bigwedge^{k} E$, as $m_{*} \notin T_{n^{\prime}, m^{\prime}}$ in general. (For instance take $m=6, n=4, k=$ 3.)

Remark 3.6. For applications of subbundles, one often fixes a choice of $d \in T$. Proposition 3.5 indicates that there is an advantage in selecting $d=m^{*}$. For example, given an $\mathcal{L}^{m^{*}}$-twisted subbundle $F \subset E$, its $\operatorname{determinant} \operatorname{det} F$ is then a twisted line bundle over the gerbe $\mathcal{S}^{m^{*}}$. This is consistent with $\operatorname{det} F \subset \wedge^{n} E$ being an $\mathcal{S}^{\left(m^{\prime}\right)^{*}}$-twisted line subbundle, where $\left(m^{\prime}\right)^{*}=m^{\prime}=\operatorname{rank}\left(\bigwedge^{n} E\right)=m^{*}$.

One can easily interpret $\mathcal{L}^{d}$-twisted subbundles using twisted homomorphisms of Definition 3.2

Proposition 3.7. Suppose $F \subset E$ is an $\mathcal{L}^{d}$-twisted subbundle.

1) Then $F$ is the same as an injective twisted homomorphism $f: F \rightarrow E$, which satisfies $f_{j}=f_{i} \otimes \chi_{i j}$ on $F_{j}=F_{i} \otimes L_{i j}^{d}$, where $\chi_{i j}: L_{i j}^{d} \rightarrow L_{i j}$ maps the basis $\xi_{i j}^{d}$ to the basis $\xi_{i j}$.

2) Assume the defining cycle $\underline{z}$ of $\mathcal{L}$ is of order $m$ so that $L_{i}$ carries a basis $e_{i}$ with $e_{j}=e_{i} \otimes \xi_{i j}^{d}$ by Proposition 1.1. Then $F$ is objective in the sense that $f$ is a $g$-objective homomorphism, where $g=\left\{g_{i}: L_{i}^{n} \rightarrow L_{i}^{n d}\right\}$ and $g_{i}$ maps the basis $e_{i}^{d}$ to $e_{i}^{n d}$.

Proof. 1) This portion follows easily from $F \prec \mathcal{L}^{d}$.

2) Since $F \prec \mathcal{L}^{d}$, we have $\mathcal{K}=\mathcal{L}^{d}$ and $K=L^{k}$ in the notation of Definition 3.2 . where $n d=m k$. Hence $g$ here maps $L^{n}$ to $K^{m}=L^{k m}=L^{n d}$. 
Note that $f, g$ depend on the choices of the trivializations (bases) $\left\{\xi_{i j}, e_{i}\right\}$. When $E=\left\{E_{i}\right\}$ is a collection of trivial bundles, there is an associated collection of bases $\left\{e_{i}\right\}$ on $L=\left\{\operatorname{det} E_{i}\right\} \prec \mathcal{L}^{m}$.

Twisted subbundles are a subtle issue but have been neglected somewhat in the literature so far. One should find both Definitions 3.2 and 3.4 useful in different contexts and can feel free to switch between them accordingly.

\section{Objective Hermitian-Einstein metrics and stability}

A Hermitian gerbe metric $\mathcal{H}=\left\{h_{i j}\right\}$ on $\mathcal{L}=\left\{L_{i j}\right\}$ is any family of fiber metrics $h_{i j}$ on $L_{i j}$ such that all trivializations $s_{i j k}$ of $L_{i k j}$ have norm 1 under the induced product metrics $h_{i j k}$.

Now take $(X, \Phi)$ to be a Kähler manifold of complex dimension $n$. Fix a holomorphic gerbe $\mathcal{L}$, namely all $L_{i j}$ and $s_{i j k}$ are holomorphic. In this case the gerbe class $\left[z_{i j k}\right]$ lives in $H^{2}\left(X, \mathcal{O}^{*}\right)$, the sheaf cohomology of non-vanishing holomorphic functions. Then there is a unique connection $\mathcal{A}=\left\{A_{i j}\right\}$ on $\mathcal{L}$ such that each $A_{i j}$ is compatible with $h_{i j}$ and the holomorphic structure on $L_{i j}$ in the usual sense. Suggested by the curvature of $\mathcal{A}$, we say further that a B-field $B=\left\{B_{i}\right\}$ is compatible with $\mathcal{H}$ and the holomorphic structure on $\mathcal{L}$ if each $B_{i}$ is purely imaginary and $B_{i}^{0,2}=0$, respectively. Thus $B_{i}$ must be purely imaginary $(1,1)$-forms. However, there are infinitely many compatible B-fields, unlike the case of a unique compatible connection $\mathcal{A}$.

Choose a compatible B-field $B \in \Omega^{1,1} \cap \mathbf{i} \Omega^{2}$. Let $E \prec \mathcal{L}$ be a twisted vector bundle of rank $m$ with a Hermitian metric (which restricts to $\mathcal{H}$ on $\mathcal{L}$ ).

Definition 4.1. Suppose $D \prec \mathcal{A}$ is a $(1,1)$-Hermitian connection on $E$, namely all its curvature $F_{D_{i}} \in \Omega^{1,1}\left(\operatorname{End}\left(E_{i}\right)\right)$. Then $D$ is called B-twisted Hermitian-YangMills if its curvature satisfies

$$
\mathbf{i} \Lambda \widetilde{F}_{D}=\mathbf{i} \Lambda\left(F_{D}-B I\right)=c I
$$

for some real constant $c$ on $X$. Here $\Lambda \widetilde{F}_{D}=\widetilde{F}_{D} \cdot \Phi$ is the usual projection $\Omega^{1,1} \rightarrow \Omega^{0}$ along the direction of $\Phi \in \Omega^{1,1}$.

Let us do some preliminary analysis of the nature of equation (4). Since $B I$ is diagonal with equal entries, (44) splits into a pair of equations:

$$
\Lambda F_{D}=\frac{\operatorname{tr}\left(\Lambda F_{D}\right)}{m} I, \quad \mathbf{i} \operatorname{tr}\left(\Lambda F_{D}\right)-\mathbf{i} m \Lambda B=m c .
$$

By taking a refinement cover we may further assume $D$ to be an $\mathrm{SU}(m)$-connection. Then (5) in turn reduces to equations

$$
\Lambda F_{D}=0, \Lambda B=\mathbf{i} c .
$$

In fact only the first equation is essential, since $B$ is already fixed at the beginning and now it also needs to satisfy the second equation (which just means $B_{i}=\left.\mathbf{i} c \Phi\right|_{U_{i}}$ ). More plainly, the first equation in (6) is elliptic on each open set $U_{i}$, despite the fact that it is not well defined on $X$ since the restrictions $\Lambda F_{D_{i}}=0, \Lambda F_{D_{j}}=0$ do not match on $U_{i} \cap U_{j}$. The second algebraic equation in $B$ plays only an auxiliary role. It is introduced to compensate the first equation so that together they compose the globally well-defined equation in (4). In other words, (4) amounts to solving the same kind of local elliptic differential equations $\Lambda F_{D_{i}}=0$ as in the original gauge theory, once we choose one compatible B-field as a solution of $\Lambda B=\mathbf{i} c$. 
In order to make use of the objective Chern class in Definition 2.2. we need to introduce the objective version of the concept: suppose $L \prec \mathcal{L}^{m}$ is a holomorphic trivialization carrying a compatible Hermitian connection $A$. (The holomorphic gerbe class $[\mathcal{L}] \in H^{2}\left(X, \mathcal{O}^{*}\right)$ is an $m$-torsion, for example using the holomorphic twisted line bundle $\operatorname{det} E$.) Set $B=\frac{1}{m} F_{A}$. Since $B$ consists of $(1,1)$-purely imaginary forms, it is a B-field compatible with the Hermitian metric and holomorphic structure on $\mathcal{L}$. In this special case we say $D$ is A-objective Hermitian-Yang-Mills. Thus we have $\Lambda \widetilde{F}_{D}=\Lambda\left(F_{D}-\frac{1}{m} F_{A}\right)=c I$ and $c$ is given specifically as

$$
c=\frac{2 n \pi}{n ! \operatorname{vol}(\mathrm{X})} \frac{\operatorname{deg}_{L}(E)}{m}
$$

in terms of the $L$-twisted degree $\operatorname{deg}_{L}(E):=\int c_{1}^{L}(E) \Phi^{n-1}$.

Equivalently, one can switch the point of view by using metrics in place of connections:

Definition 4.2. Let $L \prec \mathcal{L}^{m}$ be a holomorphic trivialization and $\mathcal{H}^{m}$ be objective by a Hermitian metric $H$ on $L$. Suppose $E \prec \mathcal{L}$ is a twisted holomorphic bundle with a Hermitian metric $h \prec \mathcal{H}$. Then $h$ is called $H$-objective Einstein if the twisted curvature of the unique associated connection of $h$ satisfies

$$
\mathbf{i} \Lambda \widetilde{F}_{h}=\mathbf{i} \Lambda\left(F_{h}-\frac{1}{m} F_{H} I\right)=c I .
$$

Here $F_{H}$ is the curvature of the $H$-compatible connection on $L$; the Einstein constant factor $c$ of $E$ (with respect to $L$ ) is given by (7).

Remark 4.3. Since we don't assume the associated connection of $h$ to be $\mathrm{SU}(m)$, we will not attempt to solve the two equations in (6) separately with $B=\frac{1}{m} F_{H}$. Instead, we fix $H$ as above and consider the consequences when the single equation (8) does have a solution for $h$. As a matter of fact it will prove quite useful to combine $h, H$ together and to view them as a single metric locally in a small neighborhood of any point.

Naturally $E$ is said to be objective Hermitian-Einstein if it admits an objective Einstein metric with respect to some $H$ and $L$. A few basic facts are recorded here for later use.

Proposition 4.4. 1) Every twisted line bundle $K$ is objective Hermitian-Einstein.

2) If $E, E^{\prime}$ are objective Hermitian-Einstein with constant factors $c, c^{\prime}$, then so are $E^{*}, \bigwedge^{r} E, E \otimes E^{\prime}$, with factors $-c, r c, c+c^{\prime}$, respectively.

3) Assume $E, E^{\prime}$ are twisted over the same gerbe. Then $E \oplus E^{\prime}$ is objective Hermitian-Einstein iff $E, E^{\prime}$ are so with equal constant factors.

Proof. It is straightforward to check the statements. For future reference, let us just indicate the trivializations used to compute the various constant factors. For convenience set $\varphi=\frac{2 n \pi}{n ! \operatorname{vol}(\mathrm{X})}$, which is a value depending on the Kähler metric $\Phi$.

1) Suppose $K \prec \mathcal{L}$ with a trivialization $L \prec \mathcal{L}$. Then the Einstein constant is $\varphi \operatorname{deg}_{L}(K)=\varphi c_{1}(K \ominus L)$.

2) Suppose $E \prec \mathcal{L}, E^{\prime} \prec \mathcal{L}^{\prime}$ are twisted bundles of ranks $m, m^{\prime}$. Choose trivializations $L \prec \mathcal{L}^{m}, L^{\prime} \prec \mathcal{L}^{\prime m^{\prime}}$. Then we have $E^{*} \prec \mathcal{L}^{-1}, \bigwedge^{r} E \prec \mathcal{L}^{r}, E \otimes E^{\prime} \prec \mathcal{L} \otimes \mathcal{L}^{\prime}$, and their corresponding trivializations are $L^{-1}, L^{w}, L^{m^{\prime}} \otimes L^{\prime m}$, where $w$ denotes 
$C(m-1, r-1)$. The Einstein constants of $E^{*}, \bigwedge^{r} E, E \otimes E^{\prime}$ are respectively given as

$$
-\varphi \operatorname{deg}_{L}(E) / m, r \varphi \operatorname{deg}_{L}(E) / m, \varphi\left[\operatorname{deg}_{L}(E) / m+\operatorname{deg}_{L^{\prime}}\left(E^{\prime}\right) / m^{\prime}\right] .
$$

3) Here $E \prec \mathcal{L}, E^{\prime} \prec \mathcal{L}$, and also $E \oplus E^{\prime} \prec \mathcal{L}$. However, we may have totally different trivializations $L \prec \mathcal{L}^{m}, L^{\prime} \prec \mathcal{L}^{m^{\prime}}$; after all $E, E^{\prime}$ may have different ranks $m, m^{\prime}$ in the first place. Then $E \oplus E^{\prime}$ inherits the trivialization $L \otimes L^{\prime} \prec \mathcal{L}^{m+m^{\prime}}$. The condition on their Einstein constants means that $\operatorname{deg}_{L}(E) / m=\operatorname{deg}_{L^{\prime}}\left(E^{\prime}\right) / m^{\prime}$. Then the Einstein constant of $E \oplus E^{\prime}$ equals $\varphi$ times this common value.

Remark 4.5. Suppose $(E, h) \prec(\mathcal{L}, \mathcal{H}),(L, H) \prec\left(\mathcal{L}^{m}, \mathcal{H}^{m}\right)$ as above and $h$ is $H$ objective Einstein. For a second trivialization $\left(L^{\prime}, H^{\prime}\right) \prec\left(\mathcal{L}^{m}, \mathcal{H}^{m}\right)$, it is simple to show that $h$ is $H^{\prime}$-objective Einstein iff the difference metric $\hat{H}=H^{\prime} \ominus H$ on $\ell=L^{\prime} \ominus$ $L$ is Einstein in the usual sense. Since the holomorphic line bundle $\ell$ always admits an Einstein metric, $E$ being objective Hermitian-Einstein is essentially independent of the choice of a trivialization $L \prec \mathcal{L}^{m}$ (with a suitable choice of metrics on $L$ ).

Next we consider the algebro-geometric counterpart.

Definition 4.6. Suppose $\mathcal{L}, L, E$ are as in Definition 4.2. Then $E$ is called $L$ objective stable (or just $L$-stable) if for every holomorphic $\mathcal{L}^{m w}$-twisted line subbundle $K \subset \bigwedge^{r} E, 1 \leq r<m$, we have

$$
C(m, r) \operatorname{deg}_{L^{w}}(K)<\operatorname{deg}_{L^{w}}\left(\bigwedge^{r} E\right),
$$

where $w=C(m-1, r-1)$ as in Corollary 2.4

The word "objective" is employed in the definition because of the involvement of $L$. To be sure, we have the degrees

$$
\operatorname{deg}_{L^{w}}(K)=\int c_{1}^{L^{w}}(K) \wedge \Phi^{n-1}, \operatorname{deg}_{L^{w}}\left(\bigwedge^{r} E\right)=\int c_{1}^{L^{w}}\left(\bigwedge^{r} E\right) \wedge \Phi^{n-1}
$$

Note that the same trivialization $L^{w}$ is used for both Chern classes here. By Corollary 2.4] it is possible to express (9) as

$$
\frac{\operatorname{deg}_{L^{w}}(K)}{r}<\frac{\operatorname{deg}_{L}(E)}{m} .
$$

One can also define the notion of objective semistability by replacing $<$ with $\leq$ in the formulas (9) or (10).

Proposition 4.7. Let $E \prec \mathcal{L}, Q \prec \mathcal{S}$ be twisted holomorphic bundles of ranks $m, 1$ respectively. Then:

1) $Q$ is objective stable.

2) $E$ is objective stable iff $E^{*}$ is objective stable.

3) $E$ is $L$-objective stable iff $E \otimes Q$ is $L \otimes Q^{m}$-objective stable.

4) Suppose $E^{\prime} \prec \mathcal{L}$ is another twisted bundle with the slope equal to that $E$. If $E, E^{\prime}$ are both L-semistable, then $E \oplus E^{\prime}$ is L-semistable. (But it cannot be L-stable unless their degrees are zero.)

Proof. All statements are generalizations of the standard stability and can be proved in a similar fashion.

1) This is evident since $\bigwedge^{1} Q=Q$ does not have any non-trivial proper subtwisted bundle. 
2) As in the usual case, one can use quotient twisted bundles (sheaves) and a short exact sequence to prove the statement.

3) Note $\bigwedge^{r}(E \otimes Q)=\bigwedge^{r} E \otimes Q^{r}$ and any sub-twisted bundle $K \subset \bigwedge^{r} E \otimes Q^{r}$ can be written uniquely as $K=K^{\prime} \otimes Q^{r}$ for some subbundle $K^{\prime} \subset \wedge^{r} E$, since $Q^{r}$ is a twisted line bundle.

4) Of course $E \oplus E^{\prime} \prec \mathcal{L}$. Here any $K \subset \bigwedge^{r}\left(E \oplus E^{\prime}\right)=\bigwedge^{r} E \oplus \wedge^{r} E^{\prime}$ has a unique decomposition $K^{\prime \prime} \oplus K^{\prime}$. The rest of the proof is clear by working componentwise.

The $L$-stability of a bundle does depend on the choice of the trivialization $L$ up to a point. More precisely we have the following:

Proposition 4.8. Suppose $E \prec \mathcal{L}$ and $L, L^{\prime} \prec \mathcal{L}^{m}$ with difference bundle $\ell=$ $L \ominus L^{\prime}$.

1) If $E$ is L-stable and $\operatorname{deg}(\ell)=\int c_{1}(\ell) \wedge \Phi^{n-1} \leq 0$, then $E$ is also $L^{\prime}$-stable.

2) Let $\mathcal{S}$ be a gerbe given by $\sqrt[m]{\ell^{-1}}$ (see Example [2.6) and $Q \prec \mathcal{S}$ the associated trivialization. Then $E$ is L-stable iff $E \otimes Q$ is $L^{\prime}$-stable.

3) When the gerbe (class) $\mathcal{L}$ is trivial, the objective stability corresponds to the standard stability.

Proof. 1) Let $K \subset \bigwedge^{r} E$ be any $\mathcal{L}^{m w}$-twisted line subbundle. By Corollary 2.4. $\operatorname{deg}_{L^{\prime}}(K)=\operatorname{deg}_{L}(K)+w \operatorname{deg}(\ell)$ and $\operatorname{deg}_{L^{\prime}}\left(\wedge^{r} E\right)=\operatorname{deg}_{L}\left(\wedge^{r} E\right)+w \operatorname{deg}(\ell)$. The results follows easily.

2) One just needs to note that $Q^{m}$ is identified naturally with $\ell^{-1}$. Then by Proposition 4.7, $E$ is $L$-stable iff $E \otimes Q$ is $L \otimes Q^{m}=L^{\prime}$-stable.

3) By assumption, there is a trivialization $J \prec \mathcal{L}^{-1}$. Thus $W:=E_{i} \otimes J_{i}$ is a global holomorphic bundle. Then one shows, via Proposition 4.7, that $E$ is objective stable iff $W$ is stable in the usual sense. (The usual stability condition actually involves all proper coherent subsheaves $V \subset W$ of arbitrary ranks $r$, from which $\operatorname{det} V \subset \wedge^{r} E$. Then one needs to apply the familiar fact that any reflexive rank 1 sheaf is locally free, i.e. a line bundle. Compare also with the Appendix of [7.)

\section{Stability of objective Hermitian-Einstein bundles}

Here we establish one direction of the generalized Hitchin-Kobayashi correspondence where every objective Hermitian-Einstein bundle is objective stable. More precisely we have the following result. We follow the proof of Lübke [16] closely as described by Chapter V of Kobayashi [14].

Theorem 5.1. Consider a rank m-holomorphic twisted bundle $E \prec \mathcal{L}$ together with a trivialization $L$ of $\mathcal{L}^{m}$. If there is a Hermitian metric $H$ on $L$ such that $E$ admits an $H$-objective Einstein metric, then $E$ is L-twisted semistable. Moreover, $E=E^{1} \oplus E^{2} \oplus \cdots \oplus E^{k}$, where $E^{1}, \cdots, E^{k}$ are all twisted bundles over $\mathcal{L}$, objective stable, and objective Hermitian-Einstein with equal constant factors.

Proof. Take any $\mathcal{L}^{m w}$-twisted holomorphic line subbundle $K \subset \bigwedge^{r} E$ for each $r$ such that $1 \leq r<m$. In view of (7), the semistable version of (10) says that to show $E$ is $L$-twisted semistable, we need to prove that

$$
r c-c^{\prime} \geq 0
$$


always holds. Here $c, c^{\prime}$ are, respectively, the constant factors of the $H$-objective Einstein metrics on $E$ and $K$.

The inclusion $K \subset \bigwedge^{r} E$ yields a nowhere vanishing twisted section $s=\left\{s_{i}\right\}$ of $\hat{E}=\bigwedge^{r} E \otimes K^{*} \prec \hat{\mathcal{L}}$, where $\hat{\mathcal{L}}=\mathcal{L}^{r} \otimes \mathcal{L}^{-m w}$. Set $\hat{L} \prec \hat{\mathcal{L}}^{\hat{m}}$ to be the trivialization induced by $L$, where $\hat{m}=C(m, r)$ is the rank of $\hat{E}$. By taking a refinement cover if necessary, we can assume $s$ to be objective (see Definition [3.1). So there are nowhere vanishing local holomorphic sections $\xi=\left\{\xi_{i j}\right\}$ of $\hat{\mathcal{L}}$ and $t=\left\{t_{i}\right\}$ of $\hat{L}$ such that

$$
s \prec \xi, t \prec \xi^{\hat{m}} .
$$

(Here we adjust the notation $t$ slightly, based on the fact that the holomorphic gerbe $\hat{\mathcal{L}}$ is of order $\hat{m}$.)

Let $\hat{H}$ be the metric on $\hat{L}$, that is, induced by $H$. By Proposition 4.4, $\hat{E}$ carries an $\hat{H}$-objective Einstein metric $\hat{h}$ with the constant factor $r c-c^{\prime}$. Define a family of local functions $f=\left\{f_{i}\right\}$ on our manifold $X$, where each

$$
f_{i}=\left\|s_{i}\right\|_{\hat{h}}^{2} / \sqrt[\hat{m}]{\left\|t_{i}\right\|_{\hat{H}}^{2}}
$$

Note that $\hat{h} \prec \hat{\mathcal{H}}, \hat{H} \prec \hat{\mathcal{H}}^{\hat{m}}$, where $\hat{\mathcal{H}}$ is the gerbe metric on $\hat{\mathcal{L}}$. This and relations (12) together yield the key fact that $f$ is a globally well-defined (smooth) function on $X$. Thus $f$ must have a maximum $q$ on $X$.

Take any point $x_{0} \in f^{-1}(q)$ and a coordinate neighborhood $V$. Introduce the functional on $V$,

$$
J(f)=\sum_{\alpha, \beta} g^{\alpha \bar{\beta}} \frac{\partial^{2} f}{\partial z^{\alpha} \partial \bar{z}^{\beta}},
$$

where $g^{\alpha \bar{\beta}}$ are the components of the metric $\Phi$. Now choose $V$ so small that $V \subset U_{i}$ for some $i$ and $\sqrt[\hat{m}]{t_{i}}$ exists as a holomorphic section of $\sqrt[\hat{m}]{\hat{L}_{i}}$ on $V$. Thus we have a nowhere vanishing holomorphic section on $V$,

$$
\check{s}=s_{i} \otimes\left(t_{i}\right)^{-1 / \hat{m}} \in \Gamma\left(\hat{E}_{i} \otimes \hat{L}_{i}^{-1 / \hat{m}}\right),
$$

and $f=\|\check{s}\|_{\check{h}}^{2}$, where $\check{h}=\hat{h} \otimes \hat{H}^{-1 / \hat{m}}$. Apply the standard Weitzenböck formula to the bundle $\check{E}_{i}=\hat{E}_{i} \otimes \hat{L}_{i}^{-1 / \hat{m}}$ over $V$ :

$$
J(f)=\sum_{\alpha, \beta} g^{\alpha \bar{\beta}} \frac{\partial^{2}\|\check{s}\|_{\check{h}}^{2}}{\partial z^{\alpha} \partial \bar{z}^{\beta}}=\left\|D_{\breve{h}} \check{s}\right\|_{\check{h}}^{2}-\check{R}(\check{s}, \check{s}) .
$$

Here $D_{\breve{h}}$ is the unique compatible connection associated to $\check{h}$ (recall $\check{s}$ is holomorphic) and $\check{R}$ is its mean curvature. More precisely, $\check{R}$ is the skew symmetric form corresponding to $\mathbf{i} \Lambda F_{\breve{h}} \in \operatorname{End}\left(\check{E}_{i}\right)$, where $F_{\breve{h}}$ is the curvature of $D_{\breve{h}}$ as before.

It is not hard to check that $\check{h}$ is $\check{H}$-objective Einstein with factor $r c-c^{\prime}$ by Proposition 4.4. (Any metric $H$ on $L$ is certainly $H$-objective Einstein with factor 0 as $\operatorname{deg}_{L}(L)=0$, and $\hat{H}^{-1 / \hat{m}}$ is some power of $H$ and hence also objective Einstein with factor 0.) However, by definition, $\breve{H}=\hat{H} \otimes\left(\hat{H}^{-1 / \hat{m}}\right)^{\hat{m}}=1$ is the trivial metric on the trivial twisting trivialization $\check{L}_{i}=\hat{L}_{i} \otimes\left(\hat{L}_{i}{ }^{-1 / \hat{m}}\right)^{\hat{m}}=\mathbf{C}$. Thus $\check{h}$ is Einstein in the usual sense with the same factor $r c-c^{\prime}$. This means $\mathbf{i} \Lambda F_{\breve{h}}=\left(r c-c^{\prime}\right) I$ and (13) becomes

$$
J(f)=\left\|D_{\breve{h}} \check{s}\right\|_{\check{h}}^{2}-\left(r c-c^{\prime}\right)\|\check{s}\|_{\check{h}}^{2} .
$$


We now return to the proof of (11). Suppose this were not the case but that $r c-c^{\prime}<0$. Then the formula (14) above would yield that $J(f) \geq 0$ on $V$, in fact $J(f)>0$, as $\check{s}$ never vanishes. Since $J(f)$ attains its maximum at the interior point $x_{0} \in V$, the Hopf maximum principle says that $f$ must be a constant on $V$. Hence $J(f)=0$ on $V$, which is a contradiction.

Hence $E$ is $L$-twisted semistable. Suppose $E$ is however not quite $L$-objective stable, namely $r c-c^{\prime}=0$. Then we will seek the desirable decomposition as stated in the theorem. First (14) still says $J(f)=\left\|D_{\breve{h}} \check{s}\right\|_{\check{h}}^{2} \geq 0$ on $V$, and the maximum principle again implies $f$ is a constant on $V$, which means $V \subset f^{-1}(q)$. This is done for each $x_{0} \in f^{-1}(q)$, so $f^{-1}(q)$ is open in $X$ and hence must be $X$ as it is certainly closed. In other words, $f$ is actually a constant function on $X$.

Now that $f$ is a constant function, $J(f)=0$ on $X$. To each $x \in X$, from (14), one has $D_{\breve{h}} \check{s}=0$ on a sufficiently small neighborhood $V_{x} \subset U_{i}$ for some $i$. Namely $\check{s}$ is $D_{\breve{h}}$-flat on $V_{x}$. Since we can easily arrange $t_{i}$ to be $\hat{H}_{i}$-flat (as $L_{i}$ is a line bundle), we see that $s_{i}$, namely the inclusion $K_{i} \subset \bigwedge^{r} E_{i}$, is $D_{h}$-flat on $V_{x}$. It follows that $E=E_{x}^{\prime} \oplus E_{x}^{\prime \prime}$ for some subbundles of ranks $r, m-r$ on $V_{x}$, with $E_{x}^{\prime}, E_{x}^{\prime \prime}$ both preserving the connection $D_{h}$. Namely the metric $h$ splits with respect to the decomposition: $E_{x}^{\prime} \perp E_{x}^{\prime \prime}$. As $x \in X$ varies, we obtain a refinement cover $\left\{V_{x}\right\}$ of $\left\{U_{i}\right\}$ as well as two bundles $E^{\prime}=\left\{E_{x}^{\prime}\right\}, E^{\prime \prime}=\left\{E_{x}^{\prime \prime}\right\}$, both of which are twisted over the gerbe $\mathcal{L}$ restricted to the refinement cover. To this end we have the decomposition $E=E^{\prime \prime} \oplus E^{\prime \prime}$ on $X$, in which $E^{\prime}, E^{\prime \prime} \prec \mathcal{L}$ both carry the induced Einstein metric by $h$. Repeat the same process for $E^{\prime}, E^{\prime \prime}$. After finitely many steps, we will have to stop. The final decomposition of $E$ is what is required in the statement of the theorem.

Corollary 5.2. If $E$ is also indecomposable, namely $E \neq E^{\prime} \oplus E^{\prime \prime}$ for any twisted bundles $E^{\prime}, E^{\prime \prime} \prec \mathcal{L}$ of positive ranks, then $E$ is L-objective stable.

In fact, more is true. One can weaken the assumption by requiring additionally that $E^{\prime}, E^{\prime \prime}$ are objective stable in the direct sum.

\section{Existence of objective Einstein Metrics on StABle Bundles}

Now we work on the other direction of the correspondence, following the original papers [8, 9, 25] and the expositions [24, 14, 15, 11]. As in [24, we will adapt the approach that combines 8 and 25 ] together to our situation.

Theorem 6.1. Suppose $E \prec \mathcal{L}$ is a rank $m$ holomorphic twisted bundle and $L \prec \mathcal{L}^{m}$ is a trivialization. If $E$ is $L$-objective stable, then $E$ admits an $H$-objective Einstein metric $h$ for some Hermitian metric $H$ on $L$.

Proof. Although we state the theorem for an Einstein metric $h$, it is the most convenient to combine $h, H$ together and solve the Einstein equation for the pair $\widetilde{h}=(h, H)$. Namely, we begin by introducing the set

$\mathcal{M}=\{(h, H): h, H$ are compatible twisted Hermitian metrics on $E, L\}$.

Each $h$ leads to a unique gerbe metric $\mathcal{H}$ on $\mathcal{L}$, and the compatibility means that $H \prec \mathcal{H}^{m}$. In a small neighborhood of any point, it will be essential to view $\widetilde{h} \in \mathcal{M}$ as an ordinary (untwisted) Hermitian metric $\widetilde{h}_{i}=h_{i} \otimes H_{i}{ }^{-1 / m}$ on the bundle $\widetilde{E}_{i}=E_{i} \otimes\left(\sqrt[m]{L_{i}}\right)^{*}$ (wherever existing). In this way the local picture matches that of the standard case in $[8,9,25,24$, and similar local computations, including 
pointwise algebraic operations, can be carried over to our case without essential changes. The proof can then be repeated almost verbatim. We outline below the main steps and leave the detailed checking to the interested reader.

Step 1. Define a Donaldson type functional $\mathcal{D}: \mathcal{M} \rightarrow \mathbf{R}$.

Dropping the positive definiteness requirement for $(h, H)$ in $\mathcal{M}$, we have the larger space $\mathcal{B}$ of pairs of compatible Hermitian forms on $E, L$. Here $\mathcal{B}$ is a Banach space containing $\mathcal{M}$ as a convex subset. This endows $\mathcal{M}$ with a Banach manifold structure and identifies the tangent space $T_{\widetilde{h}} \mathcal{M}=\mathcal{B}$ naturally at any point $\widetilde{h}$. Fix some $\widetilde{k}=(k, K) \in \mathcal{M}$ as a base point. Then $Q_{1}(\widetilde{h})=\left\{\log \left(\operatorname{det}\left(\widetilde{k}_{i}^{-1} \widetilde{h}_{i}\right)\right)\right\}$ is defined, since $\widetilde{k}_{i}, \widetilde{h}_{i}$ are genuine metrics pointwise and $\widetilde{k}_{i}^{-1} \widetilde{h}_{i} \in \operatorname{End}\left(\widetilde{E}_{i}\right)$. In fact $Q_{1}(\widetilde{h})$ is a globally well-defined function on $X$ by the internal compatibility of $\widetilde{k}, \widetilde{h}$ and $\operatorname{End}\left(\widetilde{E}_{i}\right)=\operatorname{End}\left(E_{i}\right)$ canonically. Let $\widetilde{h}(t)=(h(t), H(t))$ be a curve in $\mathcal{M}$ joining $\widetilde{h}$ to $\widetilde{k}$. Likewise we see that $\widetilde{h}(t)^{-1} \partial_{t} \widetilde{h}(t) \in \Omega^{0}(\operatorname{End}(E))$ is a global section, where $\partial_{t} \widetilde{h}(t)=\frac{d}{d t} \widetilde{h}(t)$. The associated connection of $\widetilde{h}_{i}$ on $\widetilde{E}_{i}$ has the curvature $F_{\widetilde{h}_{i}}$ equal to the twisted curvature $\widetilde{F}_{h_{i}}=F_{h_{i}}-\frac{1}{m} F_{H_{i}} I$ of $h$. As it has been used several times before, the collection $\left\{\widetilde{F}_{h_{i}}\right\}$ yields a global section in $\Omega^{2}(\operatorname{End}(E))$. Hence we have a global section $F_{\widetilde{h}}=\left\{F_{\widetilde{h}_{i}}\right\}$ as well, and we can introduce a 2 -form $Q_{2}(\widetilde{h})=\mathbf{i} \int_{0}^{1} \operatorname{Tr}\left[\left(\widetilde{h}(t)^{-1} \partial_{t} \widetilde{h}(t)\right) \cdot F_{\widetilde{h}(t)}\right] d t$ on $X$. Now define the functional

$$
\mathcal{D}(\widetilde{h})=\int_{X}\left[Q_{2}(\widetilde{h})-\frac{c}{n} Q_{1}(\widetilde{h}) \Phi\right] \wedge \frac{\Phi^{n-1}}{(n-1) !}
$$

with $c$ the (potential) Einstein constant given in (7). To verify $Q_{2}(\widetilde{h})$ is independent of the paths $\widetilde{h}(t)$, it is sufficient to check

$$
\oint_{c} \operatorname{Tr}\left[\left(\widetilde{h}(t)^{-1} \partial_{t} \widetilde{h}(t)\right) \cdot F_{\widetilde{h}(t)}\right] d t \in \operatorname{Im} \partial+\operatorname{Im} \bar{\partial}
$$

for any closed path $c=\widetilde{h}(t) \subset \mathcal{M}$. The latter depends on the same kind of local computations as in the standard case [8, and they can be transplanted over directly.

Step 2. Establish the main properties of the grading flows for $\mathcal{D}$.

Given two tangent vectors $v, w \in \mathcal{B}=T_{\widetilde{h}} \mathcal{M}, \widetilde{h}^{-1} v$ and $\widetilde{h}^{-1} w$ are global sections of $\operatorname{End}(E)$. The Riemann metric on $\mathcal{M}$ is defined by the inner product $(v, w)=$ $\int_{X} \operatorname{Tr}\left(\widetilde{h}^{-1} v \cdot \widetilde{h}^{-1} w\right) \Phi^{n}$. For a smooth family of $\widetilde{h}(t) \subset \mathcal{M}$, one has $\frac{d}{d t} Q_{1}(\widetilde{h}(t))=$ $\operatorname{Tr}\left(\widetilde{h}(t)^{-1} \partial_{t} \widetilde{h}(t)\right)$ and

$$
\frac{d}{d t} Q_{2}(\widetilde{h}(t))-\mathbf{i} \operatorname{Tr}\left(\widetilde{h}(t)^{-1} \partial_{t} \widetilde{h}(t) \cdot F_{\widetilde{h}(t)}\right) \in \operatorname{Im} \partial+\operatorname{Im} \bar{\partial}
$$

by the same local computations as in $[8,14$. Hence one has the variation of the functional

$$
\begin{aligned}
\frac{d}{d t} \mathcal{D}(\widetilde{h}(t)) & =\int_{X}\left[\mathbf{i} \operatorname{Tr}\left(\widetilde{h}(t)^{-1} \partial_{t} \widetilde{h}(t) \cdot F_{\widetilde{h}(t)}\right)-\frac{c}{n} \operatorname{Tr}\left(\widetilde{h}(t)^{-1} \partial_{t} \widetilde{h}(t)\right) \Phi\right] \wedge \frac{\Phi^{n-1}}{(n-1) !} \\
& =\int_{X} \operatorname{Tr}\left[\mathbf{i} \widetilde{h}(t)^{-1} \partial_{t} \widetilde{h}(t) \cdot F_{\widetilde{h}(t)} \wedge \Phi^{n-1}-\frac{c}{n} \widetilde{h}(t)^{-1} \partial_{t} \widetilde{h}(t) \Phi^{n}\right] \frac{1}{(n-1) !} \\
& =\int_{X} \operatorname{Tr}\left[\widetilde{h}(t)^{-1} \partial_{t} \widetilde{h}(t)\left(\frac{\mathbf{i}}{n} \Lambda F_{\widetilde{h}(t)} \Phi^{n}-\frac{c}{n} \Phi^{n}\right)\right] \frac{1}{(n-1) !} \\
& =\int_{X} \operatorname{Tr}\left[\widetilde{h}(t)^{-1} \partial_{t} \widetilde{h}(t) \cdot \widetilde{h}(t)^{-1} \widetilde{h}(t)\left(\mathbf{i} \Lambda F_{\widetilde{h}(t)}-c I\right)\right] \frac{\Phi^{n}}{n !} \\
& =\left(\partial_{t} \widetilde{h}(t), \widetilde{h}(t)\left(\mathbf{i} \Lambda F_{\widetilde{h}(t)}-c I\right)\right),
\end{aligned}
$$


which means the gradient vector field of $\mathcal{D}$ is $\operatorname{grad} \mathcal{D}(\widetilde{h})=\widetilde{h}\left(\mathbf{i} \Lambda F_{\widetilde{h}}-c I\right)$. In view of $\Lambda F_{\widetilde{h}}=\Lambda \widetilde{F}_{h}$ and (8), it also follows that $\widetilde{h}=(h, H)$ is a critical point of $\mathcal{D}$ iff $h$ is an $H$-objective Einstein metric.

A downward gradient flow $\widetilde{h}=\widetilde{h}(t)$ of $\mathcal{D}$ is then determined by the equation $\partial_{t} \widetilde{h}=-\widetilde{h}\left(\mathbf{i} \Lambda F_{\widetilde{h}}-c I\right)$, or in terms of the global section $s(t)=\widetilde{h}(0)^{-1} \widetilde{h}(t)$ on $\operatorname{End}(E)$,

$$
\frac{d s}{d t}=-\Delta_{0}^{\prime} s-\mathbf{i} s\left(\Lambda F_{\widetilde{h}(0)}-c I\right)+\mathbf{i} \Lambda\left(\bar{\partial}_{0} s \cdot s^{-1} \partial_{0} s\right),
$$

where the $\Delta_{0}^{\prime}, \partial_{0}, \bar{\partial}_{0}$ are the operators associated with the initial metric pair $\widetilde{h}(0)$. This is a non-linear parabolic equation of the same type as in [8] and can be solved essentially in the same way. Briefly, by linearizing the equation and the Fredholm theory one obtains the short time existence, and by a continuity argument, one has the long time existence. In the end, together with the maximum principle, one shows that starting at any $\widetilde{h}(0) \in \mathcal{M}$, the evolution equation (15) has a unique solution $\widetilde{h}(t)$ defined for all time $t$ together with a uniform bound

$$
\max _{X}\left|\Lambda F_{\widetilde{h}(t)}\right| \leq C \text {. }
$$

For the remaining proof we will set $\widetilde{h}(0)=\widetilde{k}$, so $\operatorname{det}\left(\widetilde{k}^{-1} \widetilde{h}(t)\right)=1$ at $t=0$. By the maximum principle again, we have $\operatorname{det}\left(\widetilde{k}^{-1} \widetilde{h}(t)\right)=1$ identically along the entire flow.

Step 3. Under the previous assumption prove the following key estimate:

$$
\max _{X}\left|\log \left(\widetilde{k}^{-1} \widetilde{h}(t)\right)\right| \leq C_{1}+C_{2} \mathcal{D}(\widetilde{h}(t))
$$

for all $t \geq 0$, where $C_{1}, C_{2}$ are positive constants independent of $t$.

The $L$-stability is used in this step. The estimate (17) substitutes for a weaker version in [9] for the case of projective varieties, whose proof requires a MehtaRamanathan's restriction theorem on stable bundles. Here we follow 24. closely, based on analytic results from [25. It goes very briefly as follows.

Write $u(t)=\log \left(\widetilde{k}^{-1} \widetilde{h}(t)\right) \in \Omega^{0}(\operatorname{End}(\widetilde{E}))$; this is a self-adjoint endomorphism and so has real eigenvalues only. (We point out that although $\widetilde{E}=E_{i} \otimes\left(\sqrt[m]{L_{i}}\right)^{*}$ involves the choice of the root $\sqrt[m]{L_{i}}$, the eigenvalues and the various norms of $u$ are well defined. This note should be kept in mind for the rest of the argument.) Because of (16), there are constants $A_{1}, A_{2}$ independent of $t$ such that $\max _{X}|u| \leq$ $A_{1}+A_{2}\|u\|_{L^{1}}$.

We will show (17) using proof by contradiction. Suppose it were not true. Then there are sequences of $t_{i} \rightarrow \infty$ and constants $B_{i} \rightarrow \infty$ such that

$$
\left\|u_{i}\right\|_{L^{1}}=\left\|u\left(t_{i}\right)\right\|_{L^{1}} \rightarrow \infty,\left\|u_{i}\right\|_{L^{1}} \geq B_{i} \mathcal{D}\left(\widetilde{h}\left(t_{i}\right)\right) .
$$

Renormalize $v_{i}=u_{i} /\left\|u_{i}\right\|_{L^{1}}$ so that $\left\|v_{i}\right\|_{L^{1}}=1$. Moreover, by (18), $\bar{\partial} v_{i}$ is bounded in $L^{2}$. Thus there is a subsequence $v_{i}$ weakly convergent to $v_{\infty} \in L_{1}^{2}$. The limit $v_{\infty}$ is self-adjoint almost everywhere, and it can be shown that its eigenvalues $\lambda_{1}, \cdots, \lambda_{r}$ are constants. Let $\{\gamma\}$ be the set of intervals between the eigenvalues, and for each $\gamma$ choose a function $p_{\gamma}: \mathbf{R} \rightarrow \mathbf{R}$ such that $p_{\gamma}\left(\lambda_{i}\right)=1$ for $\lambda_{i}<\gamma$ and $p_{\gamma}\left(\lambda_{i}\right)=0$ otherwise.

Define $\pi_{\gamma}=p_{\gamma}\left(v_{\infty}\right) \in L_{1}^{2}(\operatorname{End}(\widetilde{E}))$. This can be viewed as an $L_{1}^{2}$ subbundle $\widetilde{S}_{\gamma}$ of $\widetilde{E}$ through projection in the sense that $\pi_{\gamma}^{2}=\pi_{\gamma}$. By the main regularity theorem 
of [25], $\widetilde{S}_{\gamma}$ is actually a smooth subsheaf of $\widetilde{E}$. Since the proof is purely local, there is a smooth subsheaf $S_{\gamma}$ of $\left\{E_{i}\right\}$ such that $\widetilde{S}_{\gamma}=S_{\gamma} \otimes \mathcal{O}\left(\sqrt[m]{L_{i}}\right)^{*}$ locally. Taking the determinant we set

$$
K_{\gamma}=\operatorname{det} S_{\gamma}=\left(\bigwedge^{b} S_{\gamma}\right)^{* *}
$$

which is an $\mathcal{L}^{m w}$-twisted line subbundle of $\bigwedge^{b} E$, where $b=\operatorname{rank} S_{\gamma}=\operatorname{Tr}\left(\pi_{\gamma}\right)$ and $w=C(m-1, b-1)$. We will show for one of the $K_{\gamma}$,

$$
\frac{\operatorname{deg}_{L^{w}}\left(K_{\gamma}\right)}{b}=\frac{\operatorname{deg}_{L^{w}}\left(K_{\gamma}\right)}{\operatorname{Tr}\left(\pi_{\gamma}\right)} \geq \frac{\operatorname{deg}_{L}(E)}{m} .
$$

That is to say, $C(m, b) \operatorname{deg}_{L^{w}}\left(K_{\gamma}\right) \geq \operatorname{deg}_{L^{w}}\left(\bigwedge^{b} E\right)$. This however violates the $L$ stability of $E$, yielding our expected contradiction.

To show (19) let $\widetilde{k}_{\gamma}$ be the restriction of the base metric $\widetilde{k}$ to $\widetilde{S}_{\gamma}$. By direct computations, the curvature satisfies $\Lambda F_{\widetilde{k}_{\gamma}}=\pi_{\gamma} \Lambda F_{\widetilde{k}} \pi_{\gamma}+\Lambda\left(\bar{\partial} \pi_{\gamma} \partial \pi_{\gamma}\right)$ for every $\gamma$. It follows that $\operatorname{deg}_{L^{w}}\left(K_{\gamma}\right)=\mathbf{i} \int_{X} \operatorname{Tr}\left(\pi_{\gamma} \Lambda F_{\widetilde{k}}\right) \Phi^{n}-\int_{X}\left|\bar{\partial} \pi_{\gamma}\right|^{2} \Phi^{n}$. Let $a$ denote the largest eigenvalue of $v_{\infty}$ and $a_{\gamma}$ the width of the interval $\gamma$. Then $v_{\infty}=a \operatorname{Id}-\sum a_{\gamma} \pi_{\gamma}$ and a combination of degrees can be computed:

$$
\begin{aligned}
& a \operatorname{deg}_{L}(E)-\sum_{\gamma} a_{\gamma} \operatorname{deg}_{L^{w}}\left(K_{\gamma}\right) \\
& =\mathbf{i} \int_{X} \operatorname{Tr}\left(v_{\infty}\right) \Lambda F_{\widetilde{k}} \Phi^{n}+\int_{X} \sum_{\gamma} a_{\gamma}\left|\bar{\partial}\left(\pi_{\gamma}\right)\right|^{2} \Phi^{n} \\
& =\mathbf{i} \int_{X} \operatorname{Tr}\left(v_{\infty}\right) \Lambda F_{\widetilde{k}} \Phi^{n}+\int_{X}\left(\sum_{\gamma} a_{\gamma}\left(d p_{\gamma}\right)^{2}\left(v_{\infty}\right)\left(\bar{\partial} v_{\infty}\right), \bar{\partial} v_{\infty}\right) \Phi^{n} \\
& \leq 0,
\end{aligned}
$$

where the last inequality comes from Lemma 5.4 of 24. On the other hand,

$$
a \cdot m-\sum \operatorname{Tr}\left(\pi_{\gamma}\right)=\operatorname{Tr}\left(v_{\infty}\right)=0 .
$$

Together with the inequality above, this shows that (19) must hold for at least one $\gamma$.

Step 4. Existence of the objective Einstein metric as a limit metric.

Owing to the strong estimate (17), unlike [8, here we can avoid Uhlenbeck's theorems on removable singularities and Columbo gauges (which do not hold anyway in higher dimensions).

As before, set $s(t)=\widetilde{k}^{-1} \widetilde{h}(t) \in \Omega^{0}(\operatorname{End}(E))$. Then (17) translates into

$$
\max _{X}|\log (s)| \leq C_{1}+C_{2} \mathcal{D}(\widetilde{h}(t)),
$$

which yields two consequences:

(i) $\mathcal{D}$ is bounded below along $\widetilde{h}(t)$.

(ii) $\|s(t)\|_{L^{p}}$ is bounded above for each $p$.

To see (ii), just note $\mathcal{D}$ is decreasing along the downward flow $\widetilde{h}(t)$, hence $s(t)$ is bounded in $C^{0}$ - as well as $L^{p}$-norms.

From a minimizing sequence of $\mathcal{D}$ by (i) and the mean value theorem, we have a sequence $t_{i} \rightarrow \infty$ such that

$$
\frac{d}{d t} \mathcal{D}\left(\widetilde{h}\left(t_{i}\right)\right)=-\left\|\Lambda F_{\widetilde{h}\left(t_{i}\right)}-c I\right\|_{L^{2}}^{2} \rightarrow 0 .
$$

Then from the expression of $\mathcal{D}$ and noting $Q_{1}(\widetilde{h}(t))=0$ identically here, one sees that $\bar{\partial}\left(s\left(t_{i}\right)\right)$ is bounded in $L^{p}$. Together with (ii), this implies that $s\left(t_{i}\right)$ is bounded in $L_{1}^{p}$. Choose $p>n$, and by the Kondrakov compactness of $L_{1}^{p} \hookrightarrow C^{0}$ there is a 
subsequence $s\left(t_{i}\right)$ convergent to $s_{\infty}$ in $C^{0}$. Then by (16) together with the gradient equation (15) and the maximum principle, it is possible to show that $\left|\Delta s\left(t_{i}\right)\right|$ is uniformly bounded on $X$ in the sequence. (Compare the proof of Lemma 19 in [8].)

Thus for any $p, \Delta s\left(t_{i}\right)$ is bounded in $L^{p}$. By the ellipticity of the Laplace operator, this and (ii) together imply that $s\left(t_{i}\right)$ is bounded in $L_{2}^{p}$. By taking a subsequence if necessary, $s\left(t_{i}\right)$ weakly converges to $s_{\infty}$ in $L_{2}^{p}$. In other words, $\widetilde{h}\left(t_{i}\right) \rightarrow \widetilde{h}_{\infty}=\widetilde{k} s_{\infty}$ in the $L_{2}^{p}$-norm (with respect to $\widetilde{k}$ as before). Consequently, $F_{\widetilde{h}_{\infty}} \in L^{p}$ exists as a distribution and $F_{\widetilde{h}\left(t_{i}\right)} \rightarrow F_{\widetilde{h}_{\infty}}$ in $L^{p}$. In view of (20), we now have $\Lambda F_{\widetilde{h}_{\infty}}-c I=0$ weakly. The standard elliptic regularity guarantees that $\widetilde{h}_{\infty}$ must be smooth, and we have the desired $H_{\infty}$-objective Einstein metric $h_{\infty}$, which is read as $\widetilde{h}_{\infty}=\left(h_{\infty}, H_{\infty}\right)$.

\section{ACKNOWLEDGMENTS}

The author would like to thank Yongbin Ruan for several contributions and discussions that proved to be quite helpful. The early portion of the paper was written while the author was visiting IHES in the summer of 2008.

\section{REFERENCES}

[1] M. F. Atiyah, K-theory, past and present, Sitzungsberichte der Berliner Mathematischen Gesellschaft, 411-417, Berliner Math. Gesellschaft, Berlin, 2001. MR2091892

[2] P. Bouwknegt, A. L. Carey, V. Mathai, M. K. Murray, D. Stevenson, Twisted K-theory and K-theory of bundle gerbes, Comm. Math. Phys. 228 (2002) 17-45. MR1911247|(2003g:58049)

[3] P. Bouwknegt, V. Mathai, D-branes, B-fields and twisted K-theory, J. High Energy Phys. 3 (2000) 7-25. MR1756434 (2001i:81198)

[4] J.-L. Brylinski, Loop spaces, characteristic classes and geometric quantization, Progress in Mathematics 107, Birkhauser Boston, 1993. MR.1197353 (94b:57030)

[5] A. Căldăraru, Nonfine moduli spaces of sheaves on K3 surfaces, Int. Math. Res. Notice 20 (2002) 1027-1056. MR1902629 (2003b:14017)

[6] D. S. Chatterjee, On Gerbes, Ph.D. thesis, Cambridge University, 1998.

[7] R. Donagi, T. Pantev, B. A. Ovrut, R. Reinbacher, SU(4) instantons on Calabi-Yau threefolds with $\mathbb{Z}_{2} \times \mathbb{Z}_{2}$ fundamental group, J. High Energy Phys. 2004, no. 1, 022, 58 pp. MR.2045884 (2004k:14066)

[8] S. K. Donaldson, Anti self-dual Yang-Mills connections over complex algebraic surfaces and stable vector bundles, Proc. London Math. Soc. (3) 50 (1985) 1-26. MR765366 (86h:58038)

[9] Infinite determinants, stable bundles and curvature, Duke Math. J. 54 (1987) 231247. MR $885784(88 \mathrm{~g}: 32046)$

[10] _ The orientation of Yang-Mills moduli spaces and 4-manifold topology, J. Diff. Geom. 26 (1987) 397-428. MR910015 (88j:57020)

[11] S. K. Donaldson, P. B. Kronheimer, The geometry of four-manifolds, Oxford Mathematical Monographs. The Clarendon Press, Oxford University Press, New York, 1990. MR 1079726 (92a:57036)

[12] N. J. Hitchin, Lectures on special Lagrangian submanifolds, Winter School on Mirror Symmetry, Vector Bundles and Lagrangian Submanifolds (Cambridge, MA, 1999) 151-182, AMS/IP Stud. Adv. Math., 23, Amer. Math. Soc., 2001. MR1876068 (2003f:53086)

[13] D. Huybrechts, P. Stellari, Proof of Căldăraru's conjecture. In moduli spaces and arithmetic geometry, 31-42, Adv. Stud. Pure Math., 45, Math. Soc. Japan, Tokyo, 2006. MR2310257 (2008f:14035)

[14] S. Kobayashi, Differential Geometry of Complex Vector Bundles, Princeton University Press, 1987. MR909698 (89e:53100)

[15] J. Li, Anti-self-dual connections and stable vector bundles, Gauge theory and the topology of four-manifolds (Park City, UT, 1994), 23-49, IAS/Park City Math. Ser., 4, Amer. Math. Soc., Providence, RI, 1998. MR1611446 (99e:53025) 
[16] M. Lübke, Stability of Einstein-Hermitian vector bundles, Manuscripta Math. 42 (1983) 245-257. MR701206 (85e:53087)

[17] E. Lupercio, B. Uribe, Gerbes over orbifolds and twisted K-theory, Comm. Math. Phys. 245 (2004) 449-489. MR2045679 (2005m:53035)

[18] M. Mackaay, A note on the holonomy of connections in twisted bundles, Cah. Topol. Geom. Differ. Categ. 44 (2003) 39-62. MR.1961525 (2004d:53056)

[19] M. Mackaay, R. Picken, Holonomy and parallel transport for Abelian gerbes, Adv. Math. 170 (2002) 287-339. MR1932333 (2004a:53052)

[20] V. Mathai, R. B. Melrose, I. M. Singer, The index of projective families of elliptic operators, Geom. Top. 9 (2005) 341-373. MR2140985 (2005m:58044)

[21] M. K. Murray, Bundle gerbes, J. London Math. Soc. 54 (1996) 403-416. MR.1405064 (98a:55016)

[22] M. Murray, M. A. Singer, Gerbes, Clifford modules and the index theorem, Ann. Global Anal. Geom. 26 (2004), 355-367. MR2103405 (2005g:19008)

[23] J. Pan, Y. Ruan, X. Yin, Gerbes and twisted orbifold quantum cohomology, math.AG/0504369.

[24] C. T. Simpson, Constructing variations of Hodge structure using Yang-Mills theory and applications to uniformization, J. Amer. Math. Soc. 1 (1988) 867-918. MR944577(90e:58026)

[25] K. Uhlenbeck, S. T. Yau, On the existence of Hermitian-Yang-Mills connections in stable vector bundles, Comm. Pure Appl. Math. 39 (1986) S257-S293. MR861491 (88i:58154)

[26] S. Wang, A Narasimhan-Seshadri-Donaldson correspondence over non-orientable surfaces, Forum Math. 8 (1996) 461-474. MR.1393324 (98h:53043)

[27] _ Gerbes, holonomy forms and real structures, Comm. Contemp. Math. 11 (2009) 109-130. MR2498389 (2010b:53048)

[28] A. Weinstein, The Maslov gerbe, Lett. Math. Phys. 69 (2004), 3-9. MR 2104435 (2006d:53102)

[29] R. O. Wells, Differential analysis on complex manifolds, Springer Verlag, 1980. MR608414 (83f:58001)

[30] K. Yoshioka, Moduli spaces of twisted sheaves on a projective variety. In Moduli Spaces and Arithmetic Geometry, pp. 1-30, Adv. Stud. Pure Math., Vol. 45, Math. Soc. Japan, Tokyo, 2006. MR2306170 (2008f:14024)

Department of Mathematics, University of Missouri, Columbia, Missouri 65211

E-mail address: wangs@missouri.edu 